\title{
MYB transcription factor PdMYB118 directly interacts with bHLH transcription factor PdTT8 to regulate wound-induced anthocyanin biosynthesis in poplar
}

\author{
Haihai Wang ${ }^{1 \dagger}$, Xiaoqing Wang ${ }^{2 \dagger}$, Chunyan Yu ${ }^{3,4}$, Cuiting Wang ${ }^{1}$, Yanli Jin ${ }^{1,3}$ and Hongxia Zhang ${ }^{3,4^{*}}$
}

\begin{abstract}
Background: R2R3-MYB transcription factors (TFs) play important roles in plant growth and development, and response to biotic and abiotic stresses. However, their regulatory mechanisms in wound-induced anthocyanin biosynthesis in woody plants are largely unknown.

Results: In this work, we report that expression of anthocyanin biosynthesis genes (ABGs) were activated by PdMYB118, a MYB TF encoding gene from Populus deltoids, and the activation of PdMYB118 was significantly enhanced by PdTT8, a bHLH protein, through its direct interaction with PdMYB118. PdMYB118 and some ABGs were evidently induced by wound induction and methyl jasmonate (MeJA) treatment. Overexpression of PdMYB118 promoted anthocyanin accumulation in transgenic poplar upon wound induction. Furthermore, a poplar JASMONATE ZIM-domain (JAZ) protein, PtrJAZ1, repressed the transcriptional function of PdMYB118/PdTT8 complex by binding to PdTT8, and wound stimulated the biosynthesis of jasmonic acid (JA) and the degradation of PtrJAZ1.

Conclusions: Based on these observations, we proposed that PtrJAZ1 degradation triggered the expression of $A B G s$, leading to increased biosynthesis of anthocyanins in the wounded leaves of transgenic poplar. Therefore, our findings not only illustrate the crucial role of PdMYB118 in wound-induced anthocyanin biosynthesis in poplar, but also provide a molecular basis for the genetic engineering of colorful tree species.
\end{abstract}

Keywords: Anthocyanin, JAZ1, PdMYB118, PdTT8, Poplar

\section{Background}

As the major pigments, anthocyanins not only provide colors to plant flowers and fruits [1], but also function in plant resistance to different biotic and abiotic stresses [2-12]. In plants, anthocyanins are biosynthesized via

\footnotetext{
* Correspondence: hxzhang@sibs.ac.cn

${ }^{+}$Haihai Wang and Xiaoqing Wang contributed equally to this work

${ }^{3}$ College of Agriculture, Ludong University, 186 Hongqizhong Road, Yantai 264025, China

${ }^{4}$ The Key Laboratory of Molecular Module-Based Breeding of High Yield and abiotic Resistant Plants in the Universities of Shandong, Ludong University, 186 Hongqizhong Road, Yantai 264025, China

Full list of author information is available at the end of the article
}

the specific branch of flavonoid pathway [1, 13, 14]. In poplar, the biosynthesis of anthocyanins is catalized by a series of enzymes, including chalcone synthase (CHS), chalcone isomerase (CHI), flavanone 3-hydroxylase (F3H), flavanone 3'-hydroxylase (F3'H), flavonoid 3'5'-hydroxylase $\left(\mathrm{F} 3^{\prime} 5^{\prime} \mathrm{H}\right)$, dihydroflavonol 4-reductase (DFR) and anthocyanidin synthase (ANS) [7, 8]. The expression of anthocyanin biosynthetic genes $(A B G s)$ are regulated by the MBW complexes, which is composed of two kinds of transcriptional factors (TFs), R2R3-MYB and basic helixloop-helix (bHLH), and WD40-repeat proteins [15-17]. In Arabidopsis, the MBW complexes are composed of the

(c) The Author(s). 2020 Open Access This article is licensed under a Creative Commons Attribution 4.0 International License, which permits use, sharing, adaptation, distribution and reproduction in any medium or format, as long as you give appropriate credit to the original author(s) and the source, provide a link to the Creative Commons licence, and indicate if changes were made. The images or other third party material in this article are included in the article's Creative Commons licence, unless indicated otherwise in a credit line to the material. If material is not included in the article's Creative Commons licence and your intended use is not permitted by statutory regulation or exceeds the permitted use, you will need to obtain permission directly from the copyright holder. To view a copy of this licence, visit http://creativecommons.org/licenses/by/4.0/. The Creative Commons Public Domain Dedication waiver (http://creativecommons.org/publicdomain/zero/1.0/) applies to the data made available in this article, unless otherwise stated in a credit line to the data. 
R2R3-MYB factors PAP1, PAP2, MYB113 and MYB114, the bHLH factors TT8, GL3 and EGL3, and a WD40 protein TTG1 [16]. So far, similar MBW complexes have been identified in different plant species [13, 18-22].

JA is one of the essential phytohormones which plays important functions in the protection of plants from various biotic and abiotic stresses [23, 24]. JA signaling is negatively regulated by JAZs, which inhibit the expression of JA responsive genes by binding to the transcription factors such as MYC2 [25, 26]. In response to environmental stimulus, JA promotes the interaction between JAZs and the $\mathrm{SCF}^{\mathrm{COI} 1}$ ubiquitin ligase to trigger JAZ degradation via the ubiquitin/26S proteasome pathway [27]. The rapid activation of JA biosynthesis is stimulated by wound, a common response in plant development amid biotic and abiotic stresses such as insect attack, pathogen infection mechanical damage [23, 24]. In Arabidopsis, anthocyanin biosynthesis is induced by wound [28], and anthocyanin accumulation was regulated by JA signaling via the degradation of JAZ proteins to release bHLH and MYB TFs in the MBW complex [29]. In cotton, $F 3 H$ and its downstream genes in proanthocyanidins (PAs) biosynthesis were significantly induced by $V$. dahliae infection and wound [30]. In apple tree, MdWRKY40 functions as a key modulator in the wounding-induced anthocyanin biosynthesis [31].

To date, many anthocyanin associated R2R3-MYB TFs have been isolated from different plants [16, 32, 33]. However, their regulatory mechanisms in wound induced anthocyanin biosynthesis are still largely unknown. Previously, we demonstrated that PdMYB118 regulated the biosynthesis of anthocyanin in poplar [34]. In this work, we report that PdMYB118 functioned in anthocyanin biosynthesis by interacting with PdTT8 to activate the expression of $A B G s$ in poplar. Wound treatment induced JA accumulation, triggered the degradation of PtrJAZ1, and released its repression on the transcriptional activation activity of PdMYB/PdTT8 complex by directly interacting with PdTT8, and then activated the expression of $A B G s$ for anthocyanin biosynthesis.

\section{Results}

\section{PdMYB118 is involved in wound induced anthocyanin} biosynthesis in poplar

To explore the possible functions of PdMYB118 in anthocyanin biosynthesis in poplar, we previously generated transgenic Shanxin Yang plants [34]. Overexpression of PdMYB118 led to red leafed phenotype in adult transgenic plants grown in both green house and field, whereas young tissue culture plantlets showed green leaves as did the wide type (WT) (Additional file 1: Fig. S1a). When young plantlets were sub-cultured onto new MS medium, their leaves turned red and produced more anthocyanins after being cut off from their mother plants (Additional file 1: Fig. S1b-d). Therefore, wound may have prompted the biosynthesis of anthocyanin in transgenic plants. To confirm this speculation, wound induction was applied to both young leaves of tissue cultured transgenic plantlets and mature leaves of greenhouse grown transgenic plants. As we have hypothesized, both WT and transgenic leaves from tissue cultured plantlets were green, but transgenic leaf discs formed more red speckles 2 days after wound induction (Fig. 1a). Similar results were also observed in the mature leaf discs of greenhouse grown transgenic plants (Fig. 1b). Transgenic leaf discs produced more anthocyanins than did the WT control (Fig. 1c, d). These results indicate that PdMYB118 is involved in wound induced anthocyanin biosynthesis in poplar.

\section{Wound induces the expression of $A B G$ s}

Based on the observation that wound promoted anthocyanin accumulation in PdMYB118 overexpressing transgenic plants, we postulated that wound signaling may also induce the expression of $A B G s$ in wild type poplar. We found that $P d M Y B 118$ transcripts were gradually increased in the leaf discs during wound induction, and reached to the highest transcription level at $24 \mathrm{~h}$ (Fig. 2). Some ABGs, including PtrF3'H, PtrDFR2 and PtrANS1, showed similar expression pattern as PdMYB118. A responsive expression of PtrCHS1 to wound induction was also observed: it increased at $1 \mathrm{~h}$, then gradually reduced within $12 \mathrm{~h}$ and restored to the high expression level at $24 \mathrm{~h}$. PdTT8 was slight induced in wounded leaves within $3 \mathrm{~h}$, and obviously up-regulated at $24 \mathrm{~h}$. PtrCHI1 and PtrF3H were down-regulated within 3 to $12 \mathrm{~h}$ after wound induction and then reached to the normal expression level. The transcripts of PtrF3'5' $H 2$ reached a peak level within 1 $\mathrm{h}$ after wound induction and rapidly decreased to lower levels at later time points. From these data, we identified four wound-inducible ABGs: PtrCHS1, PtrF3'H, PtrDFR2 and PtrANS1.

\section{The transcriptional activation activity of PdMYB118 is enhanced by a bHLH TF PdTT8}

To clarify the exact function of $P d M Y B 118$ in wound induced anthocyanin biosynthesis, we first transiently expressed it in the leaf protoplasts of wild type Shanxin Yang plants. We found that transient expression of PdMYB118 enhanced the transcription of most ABGs, including the wound-/JA-inducibe ABGs: PtrCHS1, PtrF3'H, PtrDFR2 and PtrANS1 (Fig. 3a). In plants, anthocyanin-related MYB factors interact closely with bHLH TFs to control anthocyanin biosynthesis [15]. We cloned a poplar bHLH TF PdTT8, the homolog of MdbHLH3 in apple and TT8 in Arabidopsis, which interacted with different MYB TFs, and investigated its transcription activity. PdTT8 alone did not regulate the 

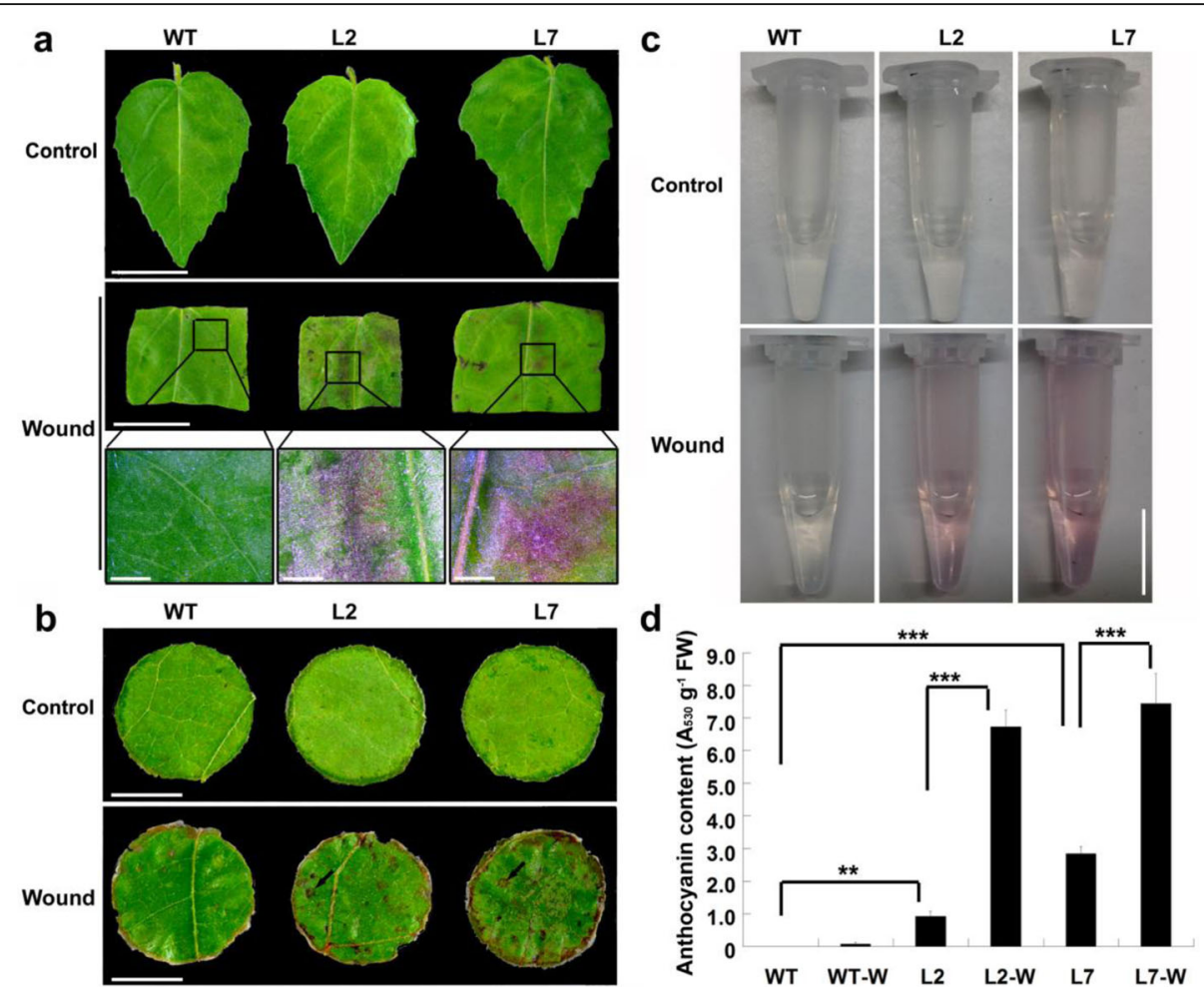

Fig. 1 Wound induced accumulation of anthocyanins in the leaves of transgenic plants overexpressing PdMYB118. a Wound prompts anthocyanin biosynthesis in the leaves of transgenic plants. The leaves of WT and transgenic plantlets cultured on MS medium were cut into pieces and transferred onto MS medium for 2 days. Images in the third row were the higher magnification of the framed area of the images in the secondary row. Scale bars for the images in the first and secondary rows stand for $1 \mathrm{~cm}$ and in the third row stand for $1 \mathrm{~mm}$. b Accumulated anthocyanin biosynthesis in the wounded leaves of transgenic plants grown in greenhouse. Matured leaves were cut into leaf discs and transferred onto MS medium for 2 days. The black arrows indicate the red speckles. Scale bar $=0.5 \mathrm{~cm}$. c Anthocyanin extracted from the wounded leaf discs in (b). Scale bar $=0.5 \mathrm{~cm}$. $\mathbf{d}$ Relative content of anthocyanins extracted from the leaves in (b). Control, before wound induction; Wound, after wound induction on MS medium for 2 days; WT, leaves of wild type Shanxin Yang plants; L2 and L7, leaves of transgenic plants overexpressing PdMYB118; WT-W, wounded leaves of wild type plant; L2-W and L7-W, wounded leaves of transgenic plants (Lines L2 and L7). Values are means and standard deviations of three biological replicates $(n=3) .{ }^{* *}$ and ${ }^{* * *}$, significant differences in comparison to WT, L2 and L7 at $P<0.01$ and $P<0.001$, respectively (Student's t-test)

expressions of $A B G s$, but could significantly enhance the transcriptional activation activity of PdMYB118. When PdTT8 was transiently co-expressed with $P d M Y B 118$ in the protoplasts, the transcription levels of all $A B G s$ were remarkably higher than those expressing $P d M Y B 118$ alone (Fig. 3a). These results imply that PdTT8 could be an efficient enhancer of PdMYB118 to regulate the expression of $A B G s$.

The enhanced transcriptional activation function of PdMYB118 by PdTT8 suggested a possible interaction of these two proteins. Therefore, we performed BiFC assays to confirm this possibility. The $\mathrm{N}$ - and $\mathrm{C}$-terminal fragment of yellow fluorescent protein (nYFP and cYFP) was fused with PdMYB118 and PdTT8, respectively. As expected, when PdMYB118-nYFP and cYFP-PdTT8 were co-transfected into poplar leaf mesophyll protoplasts, the nuclei of protoplasts showed strong YFP fluorescence; whereas no signal was detected in the control (Fig. 3b). These results demonstrate that PdTT8 could directly interact with PdMYB118 to enhance its transcriptional activation activity.

\section{Wound prompts JA biosynthesis and JAZ1 degradation}

JA biosynthesis is catalyzed by a series of biosynthetic enzymes step by step [26]. It is widely considered that JA is rapidly synthesized in plant leaves suffering wound stimulus [23, 24]. To detect the JA changes in response to wound, we examined the expressions of JA biosynthesis genes PtrAOC2-3, PtrOPR3-1, PtrACX1-2 and PtrJAR1-1. All these genes were rapidly expressed to their peak levels within $1 \mathrm{~h}$ after wound induction and then reduced gradually as the treatment elongated (Fig. 4a). The responsive expression of JA biosynthesis genes may result in JA accumulation. We then measured JA and JA-Ile contents at two time points: $1 \mathrm{~h}$ (short time) and $24 \mathrm{~h}$ (long time) after mechanical damage. JA and JA-Ile contents were about $10 \mathrm{ng} \mathrm{g}^{-1}$ and $0.58 \mathrm{ng}$ $\mathrm{g}^{-1}$ fresh weight tissues (FW) in undamaged leaves, 


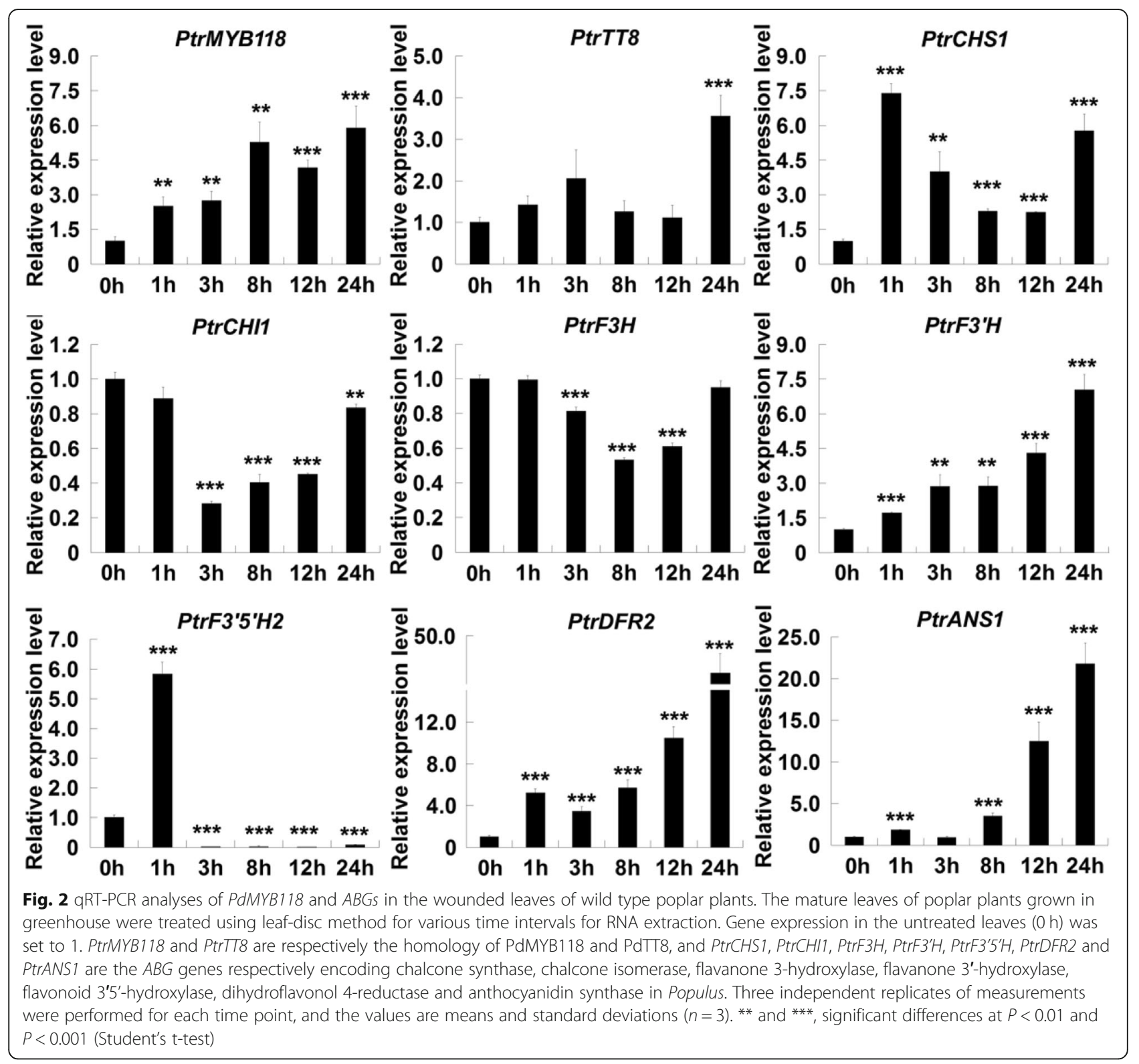

respectively. However, after $1 \mathrm{~h}$ of wound induction, the contents of JA and JA-Ile increased by about 16 and 200 folds (Fig. 4b). After 24h, JA and JA-Ile contents reduced to about 1.9 and $0.53 \mathrm{ng} \mathrm{g}^{-1} \mathrm{FW}$. The changes in JA and JA-Ile contents in wounded poplar leaves were tightly correlated with the expression changes of JA biosynthesis genes.

It has been reported that $J A Z$ genes are obviously induced by mechanical wounding [23]. We analyzed the expression of a poplar JAZ gene PtrJAZ1 during wound induction, and found that PtrJAZ1 transcripts were quickly up-regulated within $1 \mathrm{~h}$ after wound induction, rapidly decreased within $3 \mathrm{~h}$, and dropped to the lowest level after $24 \mathrm{~h}$ (Fig. 4c). The change in PtrJAZ1 protein content in response to wound induction was then tested by Western blotting. Consistent with its gene expression variation in wounded leaves, PtrJAZ1 protein accumulation reached to a higher level within $3 \mathrm{~h}$, began to decline after $8 \mathrm{~h}$, and reduced to a lower level after $12 \mathrm{~h}$ (Fig. 4d). These results indicate that wound induced anthocyanin biosynthesis in poplar may be mediated by JA signaling.

$J A$ regulates the expressions of $A B G$ s in poplar We further examined whether JA can regulate the expression of $A B G s$. Poplar leaves were sprayed with MeJA solution for qRT-PCR analyses. As expected, the expression levels of wound inducible ABGs (PtrCHS1, PtrF3'H, PtrDFR2 and PtrANS1) were up-regulated more than 3 to 20 folds by MeJA treatment (Fig. 5). JA signaling is 


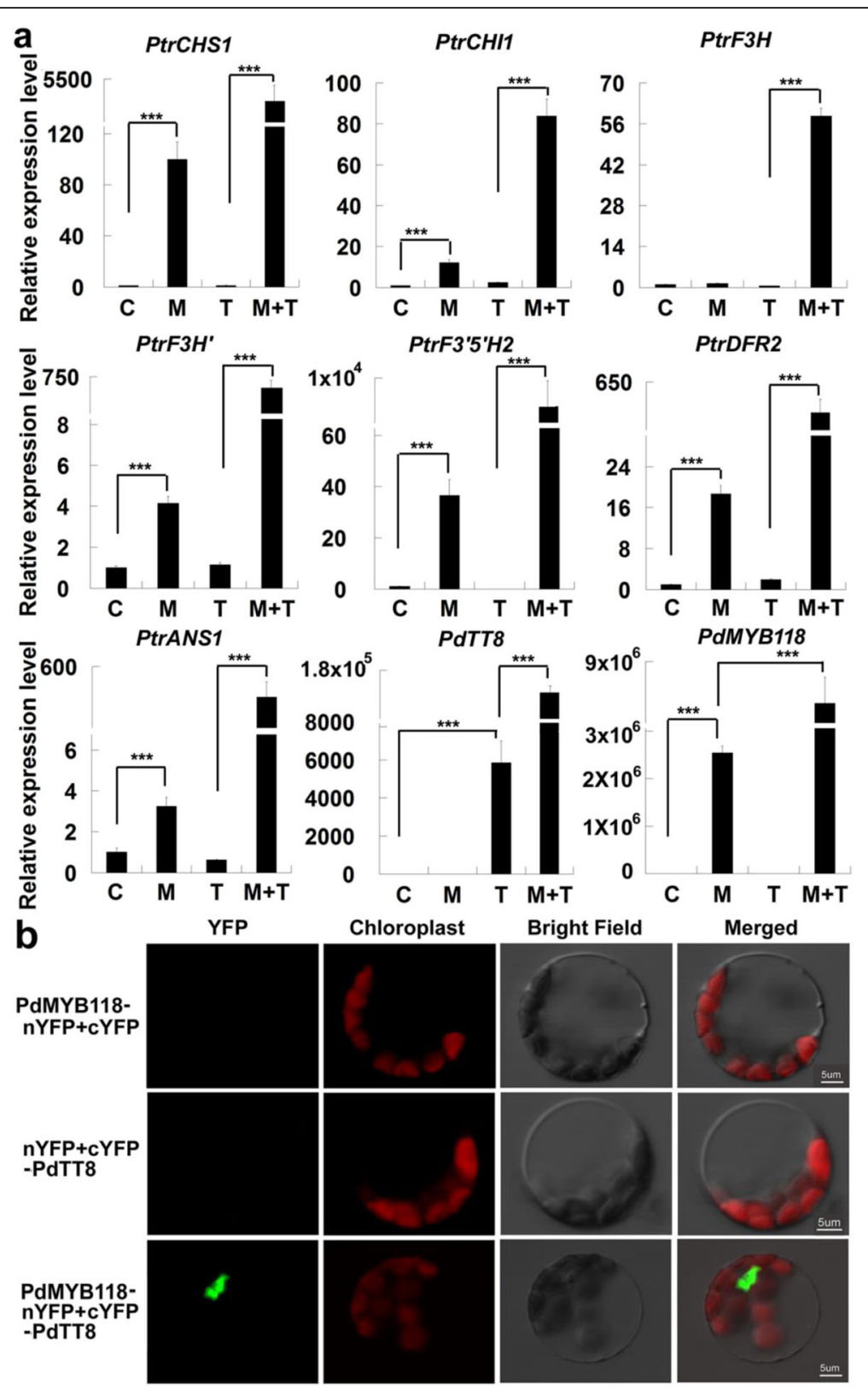

Fig. 3 PdTT8 enhances the transcriptional activation activity of PdMYB118. a Transient expression of PdMYB118 or PdTT8 in poplar mesophyll protoplasts. The constructs of pGreenll62-SK-PdMYB118, pGreenll62-SK-PdT78 or pGreenll62-SK-PdMYB118 + pGreenll62-SK-PdTT8 were transfected into the poplar leaf protoplasts, respectively. The empty vector was used as a negative control. RNA was extracted from the transfected protoplasts for qRT-PCR analyses of ABGs, PdTT8 and PdMYB118. PtrCHS1, PtrCHI1, PtrF3H, PtrF3'H, PtrF3'5'H, PtrDFR2 and PtrANS1 are the ABG genes respectively encoding chalcone synthase, chalcone isomerase, flavanone 3-hydroxylase, flavanone 3'-hydroxylase, flavonoid 3' $5^{\prime}$-hydroxylase, dihydroflavonol 4-reductase and anthocyanidin synthase in Populus. C, protoplasts transfected with pGreenll62-SK (control); M, protoplasts transfected with pGreenll62-SK-PdMYB118; T, protoplasts transfected with pGreenll62-SK-PdTT8; M + T; protoplasts transfected with pGreenll62-SKPdMYB118 and pGreenII62-SK-PdTT8. $\mathbf{b}$ BiFC assays to detect the interaction of PdTT8 and PdMYB118. PdMYB118 or PdTT8 was respectively fused with N-terminal and C-terminal fragments of YFP. Construct pairs indicated on the left were co-expressed in the leaf protoplasts of WT poplar plants. Gene expression level in the control sample was set to 1. Values are means and standard deviations of three biological replicates $(n=3)$. ${ }^{* * *}$, significant difference in comparison to $C$ and $T$ at $P<0.001$, respectively (Student's t-test)

regulated by JAZ proteins, which inhibit the expression of JA response genes by binding to other transcription factor [35]. We transiently expressed PtrJAZ1 in the leaf protoplasts of PdMYB118 transgenic plants. The up- regulated expression of most $A B G s$ by $P d M Y B 118$ was inhibited by the expression of PtrJAZ1 (Fig. 6a, b). These results indicate that JA induced expression of $A B G s$ in poplar is negatively regulated by PtrJAZ1. 

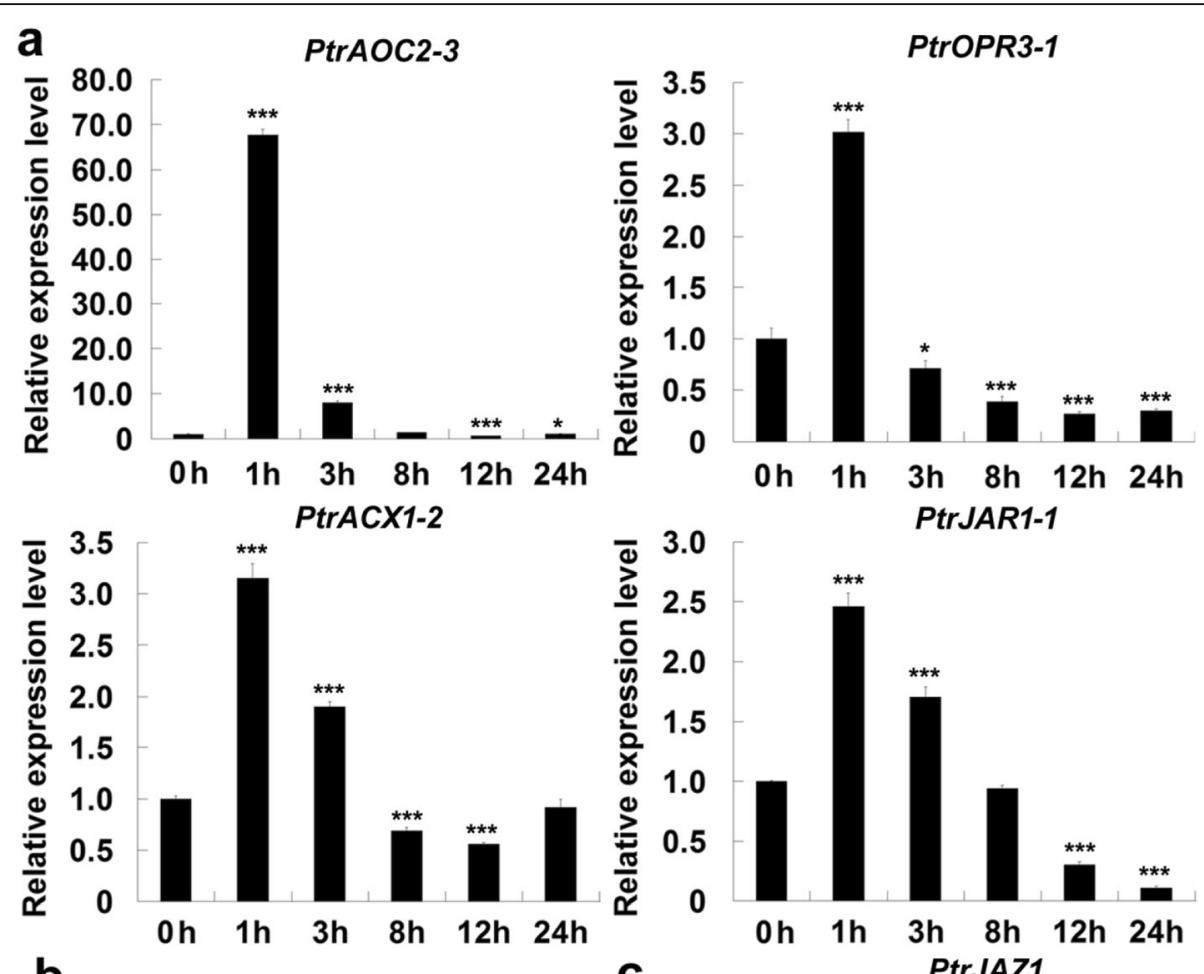

PtrJAR1-1
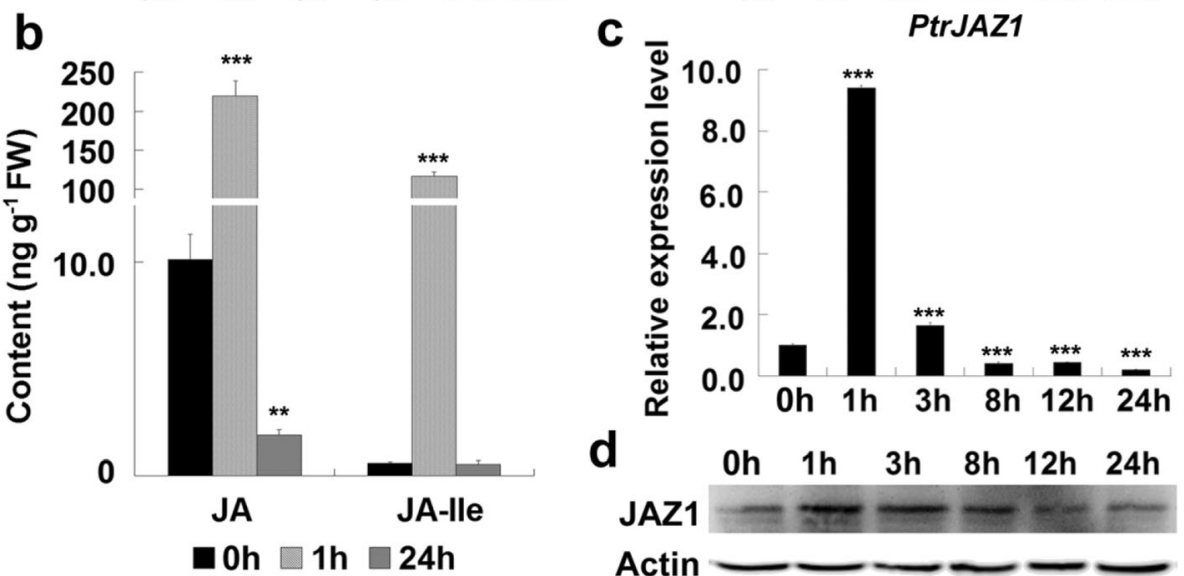

Fig. 4 JA biosynthesis gene expression, JA content and JAZ1 degradation analyses. The mature leaves of poplar plants grown in greenhouse were treated using leaf-disc method for various time intervals and then used for RNA extraction. a qRT-PCR analyses of JA biosynthesis genes in the wounded leaves. PtrAOC2-3, a putative allene oxide cyclase gene in Populus; PtrOPR3-1, a putative OPDA reductase 3 gene in Populus; PtrACX1-2, a putative acyl-CoA oxidase gene in Populus; PtrJAR1-1, a putative JASMONATE RESISTANT gene (a JA-lle biosynthesis gene) in Populus. b JA and JA-lle content in the wounded leaves of poplar plants grown in greenhouse. The leaves treated for various time intervals were used to be tested. c qRT-PCR analyses of PtrJAZ1 expression in the wounded leaves. $\mathbf{d}$ Western blotting analyses of JAZ1 protein in the wounded leaves. Total proteins were extracted from the leaf-discs treated for various time intervals. An amount of $30 \mu \mathrm{g}$ proteins were separated by $10 \%$ SDSPAGE and hybridized with Arabidopsis JAZ1 antibodies (JAZ1) or plant actin antibodies (Actin), respectively. Each gene expression in the untreated leaves $(0 \mathrm{~h})$ was set to 1 . Three independent replicates of measurements were performed for each time point, and the values are means and standard deviations $(n=3) .{ }^{*}$ and ${ }^{* *}$, significant differences at $P<0.05$ and $P<0.001$ (Student's t-test). The original uncropped blot image was shown in additional file 6: Fig. S6

PtrJAZ1 represses the transcriptional activation activity of PdMYB118/PdTT8 complex by binding to PdTT8

The suppressed transcriptional activation activity of PdMYB118 by PtrJAZ1 could be regulated by PdTT8. Therefore, we transiently expressed PdTT8 and PtrJAZ1 alone or together in the leaf protoplasts of $P d M Y B 118$ transgenic plants (Additional file 2: Fig. S2a, b). We observed that transient expression of PdTT8 in the leaf protoplasts of $P d M Y B 118$ transgenic plants further increased the expression levels of $A B G s$ (Fig. 7a). However, when PtrJAZ1 and PdTT8 were co-expressed in the leaf protoplasts of $P d M Y B 118$ transgenic plants, the 


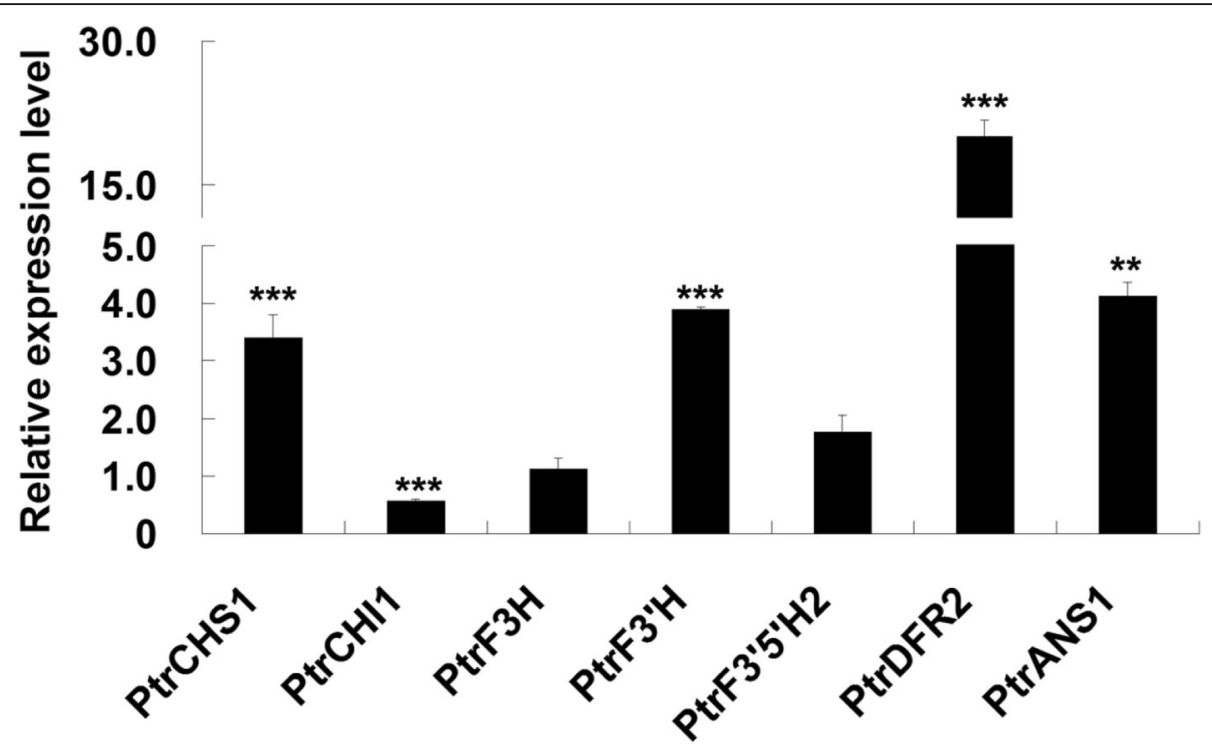

Fig. 5 gRT-PCR analyses of ABGs in MeJA treated leaves. PtrCHS1, PtrCHI1, PtrF3H, PtrF3'H, PtrF3'5'H, PtrDFR2 and PtrANS1 are the ABG genes respectively encoding chalcone synthase, chalcone isomerase, flavanone 3-hydroxylase, flavanone 3'-hydroxylase, flavonoid 3'5'-hydroxylase, dihydroflavonol 4reductase and anthocyanidin synthase in Populus. Gene expression in the leaves sprayed with water (control) was set to 1. Values are means and standard deviations of three biological replicates $(n=3) .{ }^{* *}$ and ${ }^{* *}$, significant differences at $P<0.01$ and $P<0.001$ (Student's t-test)

expression levels of $A B G s$ were repressed to the levels of control samples (Fig. 7a). These results suggest that PtrJAZ1 suppressed the transcription activity of PdMYB118/PdTT8 complex. Then, we performed BiFC assays to examine whether PtrJAZ1 interacts with PdMYB118 or PdTT8. PtrJAZ1-nYFP was transiently co-expressed with cYFP-PdTT8 or cYFP-PdMYB118 in the leaf mesophyll protoplasts of wide type poplar. Coexpression of PtrJAZ1-nYFP with cYFP-PdTT8 produced strong YFP fluorescence in the nuclei of protoplasts, whereas no fluorescence signal was detected in the protoplasts co-expressing PtrJAZ1-nYFP and cYFPPdMYB118 (Fig. 7b; Additional file 3: Fig. S3). This result is consistent with the finding in apple that MdJAZs interact with MdbHLH3 (the analogue of TT8) but not with the PA biosynthesis associated MdMYB9/11 [36].

We further confirmed the inhibition of PtrJAZ1 on the transcriptional activation activity of PdMYB118/PdTT8

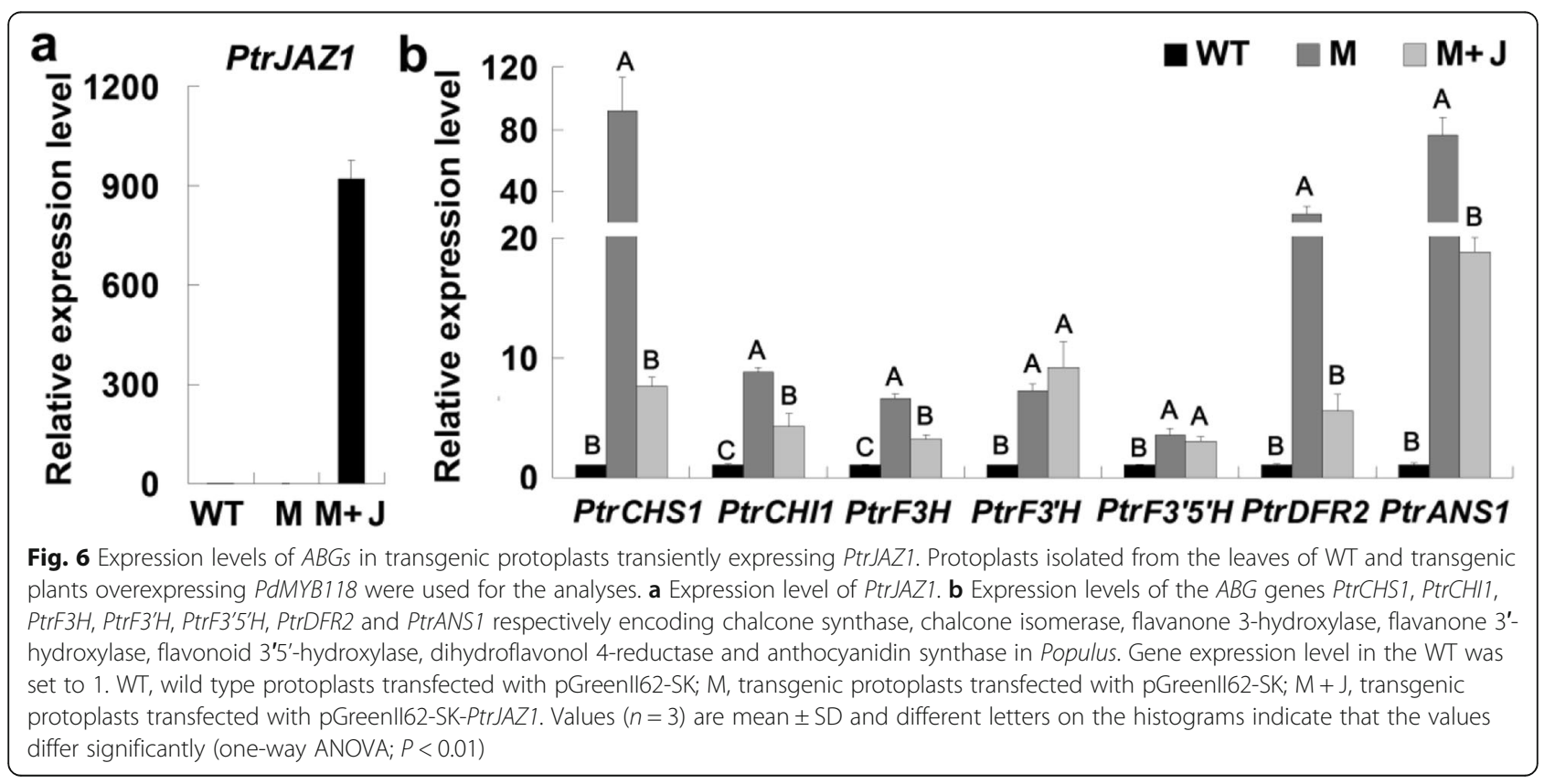



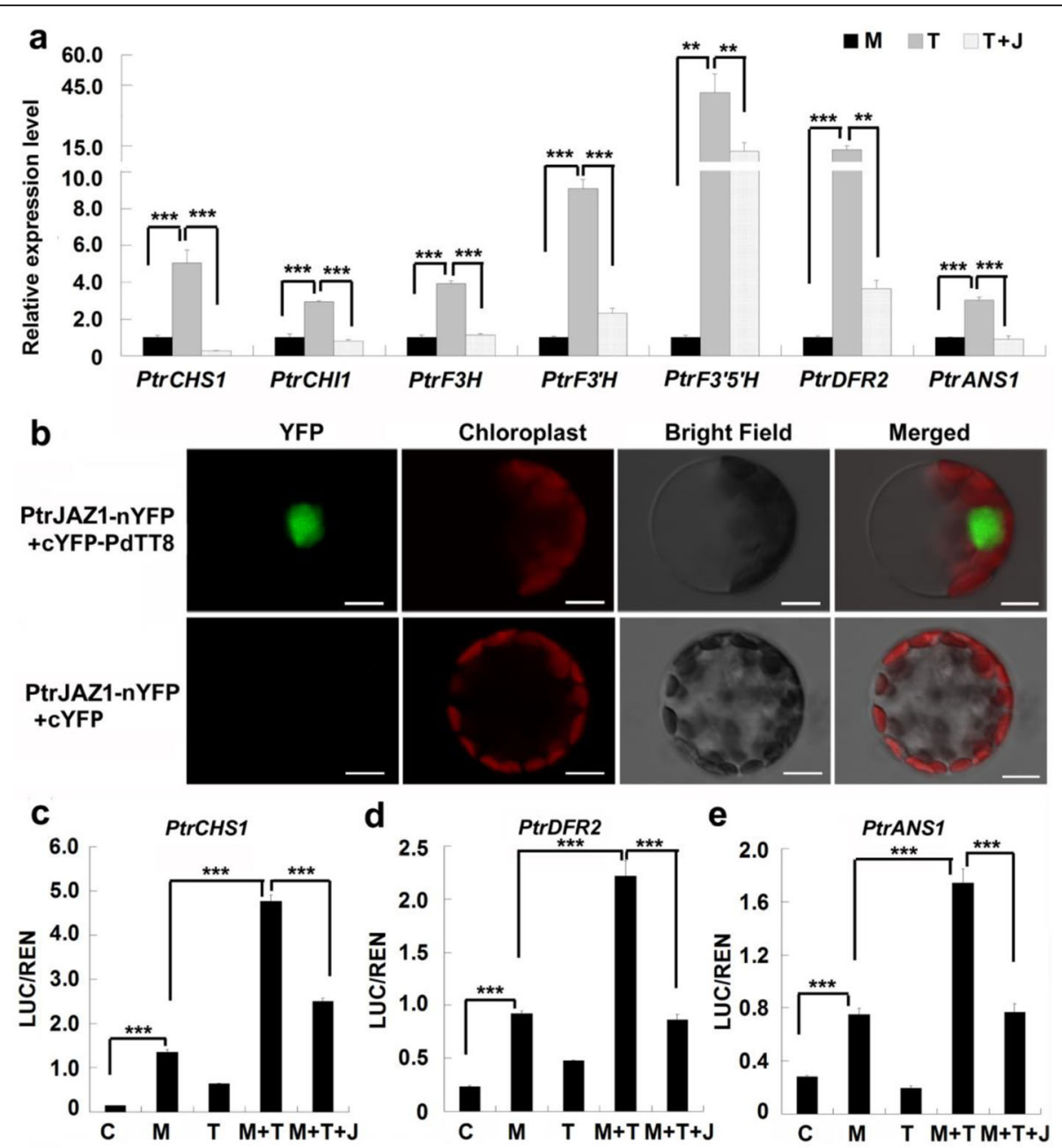

Fig. 7 PtrJAZ1 binds to PdTT8 to inhibit the transcriptional activation activity of the PdMYB118/PdTT8 complex. PtrCHS1, PtrCHI1, PtrF3H, PtrF3'H, PtrF3'5'H, PtrDFR2 and PtrANS1 are the ABG genes respectively encoding chalcone synthase, chalcone isomerase, flavanone 3-hydroxylase, flavanone 3'-hydroxylase, flavonoid 3'5'-hydroxylase, dihydroflavonol 4-reductase and anthocyanidin synthase in Populus. a PtrJAZ1 represses the transcriptional activation activity of the PdMYB118/PdTT8 complex. Protoplasts isolated from the leaves of transgenic plants overexpressing PdMYB118 were transformed with pGreenll62-SK-PdTT8 alone, or co-transformed with pGreenll62-SK-PdTT8 and pGreenll62-SK-PtrJAZ1. qRT-PCR analysis of $A B G$ s in the transfected protoplasts was performed. The empty vector was used as a negative control and the gene expression level in the control was set to 1. Values are means and standard deviations of three biological replicates $(n=3) .{ }^{* *}$ and ${ }^{* * *}$, significant differences at $P<$ 0.01 and $P<0.001$ (Student's t-test). M, transgenic protoplasts transfected with pGreenll62-SK; T, transgenic protoplasts transfected with pGreenll62-SK-PdTT8; T J, transgenic protoplasts co-transfected with pGreenII62-SK-PdTT8 and pGreenll62-SK-PtrJAZ1. $\mathbf{b}$ BiFC assays to test the interaction of PtrJAZ1 with PdTT8. PtrJAZ1 was fused with the N-terminal fragment of YFP, and PdTT8 was linked to the C-terminal fragment of YFP, respectively. Construct pairs indicated on the left were co-expressed in poplar leaf protoplasts. Scale bar $=10 \mu \mathrm{m}$. c-e Transient transcription dual-luciferase assays to show that PtrJAZ1 inhibits the transcriptional activation activity of the MYB118/TT8 complex to the promoters of PtrCHS1 (c), PtrDFR2 (d) and PtrANS1 (e). The effectors (pGreenll62-SK-PdMYB118, pGreenll62-SK-PdTT8, pGreenll62-SK-PdMYB118+ pGreenll62-SK-PdTT8 or pGreenll62-SK-PdMYB118 + pGreenll62-SK-PdTT8 + pGreenll62-SK-PtrJAZ1) and reporters (35S::REN-ProPtrCHS1::LUC, 355::REN-ProPtrDFR2::LUC or 35S::REN-ProPtrANS1::LUC) were co-expressed in poplar protoplasts. The expression level of REN was used as an internal control. The LUC/REN ratio represents relative activity of the PtrCHS1, PtrDFR2 and PtrANS1 promoters. Error bars represent the SDs from three biological replicates. C, protoplasts transfected with pGreenll62-SK (control); M, protoplasts transfected with pGreenll62-SK-PdMYB118; T, protoplasts transfected with pGreenll62-SK-PdTT8; M + T, protoplasts transfected with pGreenll62-SK-PdMYB118 and pGreenll62-SK-PdTT8; M + T + J, protoplasts transfected with pGreenll62-SK-PdMYB118, pGreenll62-SK-PdT78 and pGreenll62-SK-PtrJAZ1. Values are means and standard deviations of three biological replicates $(n=3){ }^{* * *}$, significant differences at $P<0.001$ (Student's t-test)

complex by dual-luciferase assays. Three wound inducible ABGs (PtrCHS1, PtrDFR2 and PtrANS1) were selected. The transcriptional activation activity of PdMYB118 on the promoters of PtrCHS1, PtrDFR2 and PtrANS1 was higher than that of PdTT8, showing a higher ratio of LUC/REN (Fig. 7c-e). The highest ratio values were 
observed in the protoplasts co-expressing PdMYB118 and PdTT8. And the transcription activity of PdMYB118/ PdTT8 complex decreased obviously when PtrJAZ1 was co-transformed with PdMYB118 and PdTT8 (Fig. 7c-e). These results suggest that by binding to PdTT8, PtrJAZ1 could restrain the transcriptional activation activity of PdMYB118/PdTT8 complex.

\section{Degradation of JAZ1 proteins promotes anthocyanin biosynthesis in the wounded leaves of transgenic plants} Based on the observations that expression of $A B G s$ was inhibited by PtrJAZ1 and JAZ1 proteins were degraded upon wound induction, we speculated that the increased anthocyanin biosynthesis in the wounded transgenic leaves may be a result of JAZ1 degradation. By Western blotting analysis using Arabidopsis JAZ1 antibodies, we found that JAZ1 accumulation in the wounded leaves of transgenic plants were distinctly lower than that in the no-treated control leaves (Fig. 8a). Therefore, the decreased JAZ1 protein level may have released the transcriptional activation activity of the MYB118/TT8 complex and increased the expression of wound-inducible $A B G$ s. We further analyzed the expression of $A B G s$ in the leaves of transgenic plants overexpressing $P d M Y B 118$. In the untreated leaves of transgenic plants (lines L2 and L7), wound -/JA-inducible ABGs (PtrCHS1, PtrDFR2, PtrF3H and PtrANS1) were up-regulated in transgenic plants. Upon wound induction, their expression levels increased in the leaves of both WT and transgenic plants, with a more significant increase in the wounded leaves of transgenic plants (Fig. 8b). Similarly, the expression level of PdMYB118 increased in the wounded leaves of both WT and transgenic plants, but the increase was significantly higher in transgenic plants (Additional file 4: Fig. S4a). However, the expression level of PdTT8 was only slightly changed in the leaves of both WT and transgenic leaves (Additional file 4: Fig. S4b). These results indicate that the increased anthocyanin accumulation in the wounded leaves of transgenic plants could be due to the enhanced expression of $A B G s$ resulted from the wound-induced degradation of JAZ1 proteins.

\section{Discussion}

Although wound induced anthocyanin accumulation has been widely observed in plants, the molecular mechanism that how wound regulates anthocyanin biosynthesis in poplar still remains unknown [28, 37]. Recently, we reported that $P d M Y B 118$ regulated the biosynthesis of anthocyanin in poplar [34]. Overexpression of PdMYB118 obviously activated the expression of anthocyanin biosynthesis genes but not the two proanthocyanin synthesis genes, leading to red leafed phenotype in transgenic plants. Interestingly, during the process of shoot propagation, we observed that the leaves of tissue cultured plantlets or green house grown young transgenic plants remained in green or less red color, but once the shoots were cut off, the leaves of new cut shoots turn into red color (Additional file 1: Fig. S1a-d). The stability of this phenomenon (wound-induced anthocyanin biosynthesis) was further confirmed using green leaves of both tissue cultured plantlets and greenhouse grown plants (Fig. 1a-d).

Previous studies have showed that JA regulates WDrepeat/bHLH/MYB (WBM) complex-mediated anthocyanin accumulation via the interaction of JAZ proteins with bHLH and MYB transcription factors [29]. PAspecific R2R3-MYB factors like MYB134 in poplar and MdMYB9/11 in apple dramatically responded to wound treatment, whereas the anthocyanin biosynthesis R2R3MYB factor MdMYB1 in apple did not $[8,36]$. MYB/ bHLH complex, such as PAP1/TT8 in Arabidopsis, AN4/AN1 in Petunia hybrid, VvMYBA1/VvMYC1 in grape, and MdMYB10/MdbHLH3 in apple, are also involved in anthocyanin synthesis [13, 38-40]. We found that expression of most $A B G s$ was responsive to wound treatment (Fig. 2). The poplar bHLH TF PdTT8 physically interacted with PdMYB118 to form the PdMYB118/ PdTT8 complex, and more efficiently activated the expressions of $A B G s$ (Fig. 3a, b). Therefore, PdTT8 may function as an enhancer to promote the transcriptional activation activity of PdMYB118.

Wound can rapidly activate the biosynthesis of JA, which functions as an important plant defense signal in response to various stresses $[23,24]$. In plants, the biosynthetic pathway of JA is initiated from the triunsaturated fatty acids $\alpha$-linolenic acid (18:3) [26]. After two oxidation steps, $\alpha$-linolenic acid is converted to an unstable allene oxide, which is subsequently catalyzed to 12-oxophytodienoic acid (OPDA) by an allene oxide cyclase (AOC) in chloroplasts. Then the second half of JA biosynthesis is finished in peroxisomes, where OPDA is reduced to OPC- 8 by OPDA reductase 3 (OPR3) [41] After $\beta$-oxidation, JA is transported to cytosol, where it is conjugated to amino acids by JA-amino acid synthetase (JAR1) to form jasmonoyl-isoleucine (JA-Ile), a major biologically active jasmonate among a growing number of jasmonate derivatives. We found that wound treatment rapidly up-regulated the expression of JA biosynthesis genes within $1 \mathrm{~h}$ (Fig. 4a), leading to the rapid output of JA and JA-Ile in the wounded leaves (Fig. 4b). These results indicate that JA biosynthesis responds rapidly to wound induction in poplar. Increased JA biosynthesis by wound treatment could activate the JA signaling. Indeed, similar to the expression pattern of JA biosynthesis gene, PtrJAZ1 was also up-regulated within $1 \mathrm{~h}$ after the initiation of wound induction, and then gradually decreased (Fig. 4c). This result is consistent with previous reports that most $J A Z$ genes were strongly induced within $1 \mathrm{~h}$ after wound induction and declined 


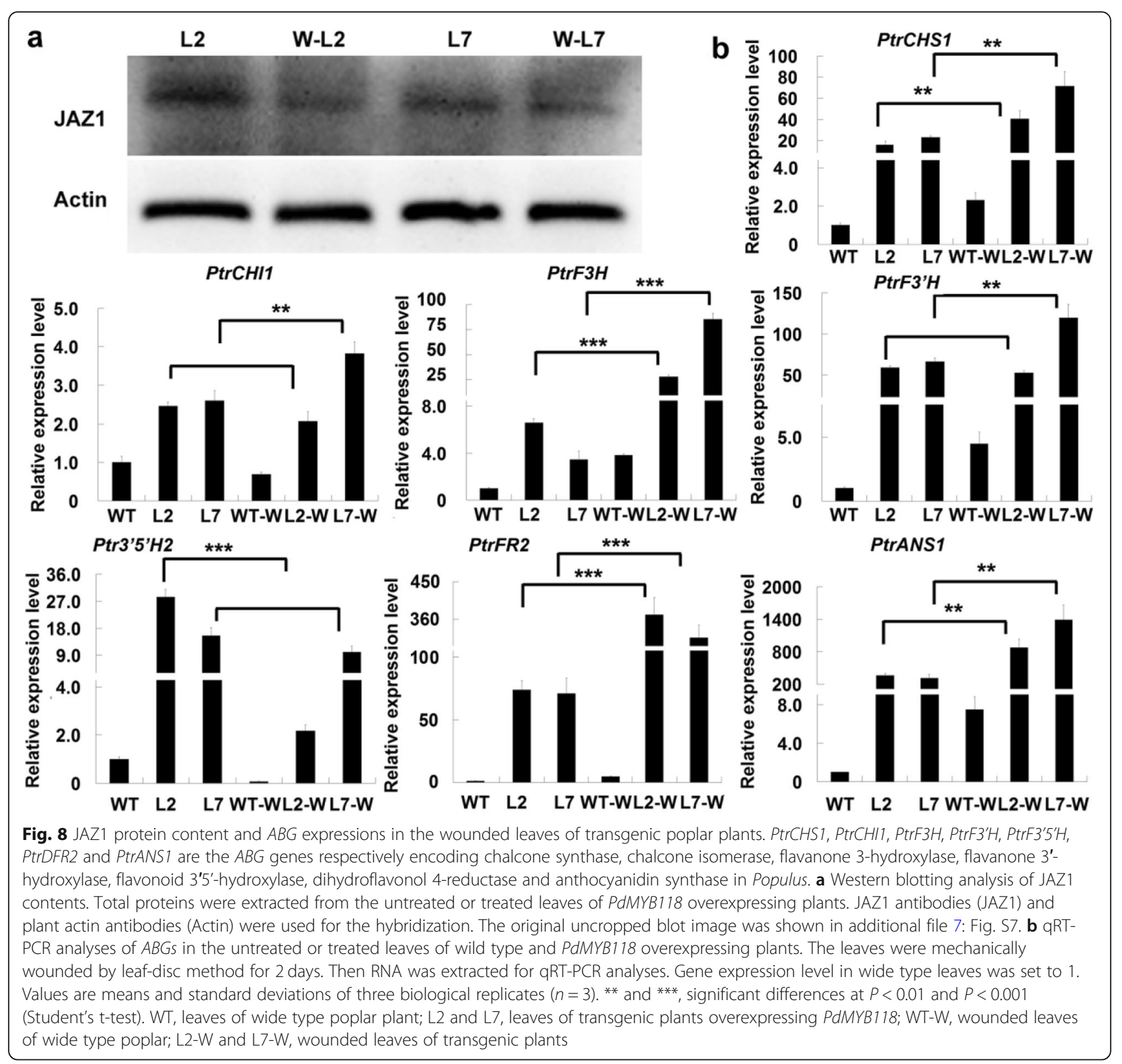

at later time points [23, 24]. JA signaling also promotes the degradation of JAZ transcriptional repressors through the activity of the E3 ubiquitin-ligase $\mathrm{SCF}^{\mathrm{COI1}}$ JAZs [27]. We found that the content of poplar JAZ proteins was also obviously decreased after $8 \mathrm{~h}$ of wound treatment (Fig. 4d). Therefore, in poplar, wound induction could rapidly induce JA biosynthesis by activating its biosynthesis genes and promote the degradation of JAZ protein.

JA regulates several anthocyanin biosynthetic genes involved in anthocyanin accumulation [42, 43]. In poplar, MeJA treatment could induce the expression of some $A B G s$ such as PtrCHS1, PtrF3H', PtrDFR2 and PtrANS1 (Fig. 5). Interestingly, expressions of these $A B G s$ were also induced by wound, indicating that wound-induced anthocyanin biosynthesis may be mediated by JA signaling. It has been reported that JA regulates WD-repeat/bHLH/ MYB complex-mediated anthocyanin accumulation via the interaction of JAZ proteins with $\mathrm{BHLH}$ and MYB factors [29]. We found that PtrJAZ1 specifically bound to PdTT8 and attenuated the transcriptional activation activity of PdMYB118/PdTT8 complex on the promoter of JA/ wound-inducible $A B G$ s (Fig. 7a-e). JAZ regulated expression of $A B G s$ in poplar is similar to that in Arabidopsis [29], but different from that in apple, where MdJAZ2 inhibits the recruitment of MdbHLH3 to the promoters of MdMYB9 and MdMYB11 which regulate the biosynthesis of anthocyanin and proanthocyanin [24]. Therefore, JA induced JAZ protein degradation could abolish the 
interaction of JAZ proteins with BHLH and MYB factors, and then activate the biosynthesis of anthocyanin [29]. In agreement with this speculation, when PtJAZ1 protein was degraded in the wounded leaves of transgenic plants, JA -/wound-inducible $A B G s$ were highly induced to promote anthocyanin biosynthesis (Fig. 8a, b).

\section{Conclusions}

Taken together, our study illustrates the biological function of PdMYB118 in wound-induced anthocyanin synthesis in poplar. Although more detailed studies are still needed to completely understand the mechanism of PdMYB118 in anthocyanin synthesis, our data presented here imply a possible model of wound-induced anthocyanin synthesis in poplar: upon wound induction, JA biosynthetic genes are rapidly activated for JA biosynthesis, subsequently triggered the degradation of PtrJAZ1; Then, the transcriptional activation activity of PdMYB118/ PdTT8 complex is restored to promote the expression of anthocyanin biosynthesis genes; Finally, anthocyanins were biosynthesized, leading to red leafed phenotype in transgenic plants (Additional file 5: Fig. S5a, b).

\section{Methods}

\section{Plant materials and growth conditions}

Wild type Shanxin Yang (WT) and transgenic Shanxin Yang $(P$. davidiana $\times P$. bolleana $)$ overexpressing $P d M Y B 118$ used in our previous report were propagated on MS medium, transferred to soil, and grown in greenhouse $[34,44]$. Shanxin Yang was provided by Prof. GuiFeng Liu (Northeast Forestry University, China). Populus deltoids and its red leaf mutant were purchased from Qingyuan HiTech Ltd. (Yantai, China). Plants were grown in greenhouse at $25^{\circ} \mathrm{C}$ (day) $/ 18^{\circ} \mathrm{C}$ (night) in a $12 \mathrm{~h}$ light/ $12 \mathrm{~h}$ dark photoperiod.

\section{Wound treatments}

For wound induction, the leaves of wild type and transgenic poplar grown on MS medium or in green house were cut into $1.5 \mathrm{~cm}$ dices and transferred onto MS medium. At various time points after wound induction, leaf discs were harvested, frozen in liquid nitrogen, and stored at $-80^{\circ} \mathrm{C}$ for RNA, protein, JA and anthocyanin extraction.

\section{MeJA treatment}

To analyze the induction of $P d M Y B 118$ and $A B G s$ by JA, $100 \mu$ M MeJA (Sigma, Shanghai, China) solution was sprayed onto the mature leaves of WT poplar plants grown in greenhouse. After $3 \mathrm{~h}$, leaves were collected and used for RNA extraction. Leaves sprayed with water were used as a negative control.

\section{Quantitative real-time RT-PCR}

Total RNA was extracted from leaves and leaf protoplasts with RNAiso Reagent (Takara, Shanghai, China) and $\mathrm{qPCR}$ was performed as described previously [45]. The relative expression of each target gene was normalized using PtrEF1 $\beta$. Gene specific primers used in this study were listed in additional file 8: Table S1. Three independent replicates of measurements were performed for each sample.

\section{Anatomical observations}

For histological observation of anthocyanin speckles, leaf dices of wild type and transgenic plants were examined with a light microscope after wound treatment for $48 \mathrm{~h}$. Images were captured under the SMZ800 microscope.

\section{Anthocyanin content determination}

After wound induction for $48 \mathrm{~h}$, anthocyanin content in the leaf discs of WT and transgenic plants grown in greenhouse was measured as described previously [46]. To detect the quantity of anthocyanin, $A_{530}$ per gram fresh weight $(\mathrm{FW})$ was used. Three replicates were carried out for each measurement, and the variability was indicated with the standard deviation (SD).

\section{Western blotting}

Western blotting analyses were performed as described previously [47]. Briefly, a total amount of $30 \mu \mathrm{g}$ proteins were extracted from the wounded leaf discs at various time points and separated by $10 \%$ sodium dodecyl sulfate-polyacrylamide gel electrophoresis (SDS-PAGE). Then total protein was electrotransferred onto polyvinylidene difluoride membranes. Immunoblotting was performed using JAZ1 antibodies against the Arabidopsis JAZ1 protein (Agrisera, http://www.agrisera.com/), then incubated with the secondary antibody goat anti-rabbit IgG-horseradish peroxidase (HRP) (Abmart, China). To detect the Actin, the primary antibody (mouse monoclonal Actin antibody) and the secondary antibody (goat anti-mouse IgG-HRP) were used to perform the immune reaction (Abmart, Shanghai, China). After incubation in the chemiluminescence detection solution LumiGLO (KPL, USA), membranes were imaged with a chemiluminescence image system Tanon 5500 (Tanon, Shanghai, China). Proteins were quantified with a BCA Protein Assay kit (Thermo, Shanghai, China).

\section{JA content assays}

For JA and JA-Ile content assays, the wounded leaves of wild type poplar plants at $0 \mathrm{~h}, 1 \mathrm{~h}$ and $24 \mathrm{~h}$ time points were ground into fine powder in liquid nitrogen, respectively. JA extraction was performed as described previously [48]. Three replicates were carried out for 
each assay, and the variability was indicated with the standard deviation (SD).

Transient expression of TFs in poplar mesophyll protoplasts To analyze the transcription function of PdMYB118, PdTT8 and PdMYB118/PdTT8 complex, CaMV 35S promoter driven transcriptional factor effectors were produced by inserting $P d M Y B 118$ or PdTT8 into pGreenII62-SK [49]. Leaf protoplasts were isolated from the leaves of Shanxin Yang as described previously [34]. The resultant constructs pGreenII62-SK-PdMYB118, pGreenII62-SK-PdTT8 and pGreenII62-SK-PdMYB118 + pGreenII62-SK-PdTT8 were transferred into protoplasts, respectively. The empty vector was used as a negative control. After kept in dark for $16 \mathrm{~h}$, the transfected protoplasts were collected for RNA extraction and qRT-PCR analyses of $A B G s$ as described above.

To detect the effects of PdTT8 or PtrJAZ1 on ABGs expression in the leaves of transgenic plants overexpressing PdMYB118, effectors pGreenII62-SK-PdTT8, pGreenII62SK-PtrJAZ1 and pGreenII62-SK-PdTT8 + pGreenII62-SKPtrJAZ1 were transformed into the mesophyll protoplasts of transgenic plants, respectively. The transfected protoplasts were used for qRT-PCR analyses of $A B G$ s. Three replicates were carried out for each assay, and the variability was indicated with the standard deviation (SD).

\section{BiFC assays}

Full-length coding sequences of PtrJAZ1, PdMYB118 and PdTT8 were individually cloned and subsequently recombined into YFP BiFC vectors so that they were fused with the $\mathrm{N}$ - or C-terminal of YFP (nYFP or cYFP) to generate pSAT4-nYFP-PdMYB118/PtrJAZ1 and pSAT4-cYFPPdTT8/PdMYB118 plasmids. Primers used for gene clone are given in Supporting Information Table S1. To detect the interaction of PtrJAZ1 with PdTT8 or PdMYB118, and the interaction of PdMYB118 with PdTT8, the relative constructs were co-transfected into the mesophyll protoplasts of wild type plants. After incubated at $23^{\circ} \mathrm{C}$ for $16 \mathrm{~h}$, the protoplasts were analyzed using a confocal microscope at $514 \mathrm{~nm}$ wavelength (Zeiss LSM 510 META). Three replicates were carried out for each assay, and the variability was indicated with the standard deviation (SD).

\section{Transient transcription dual-luciferase assays}

For dual-luciferase assays, the LUC reporter constructs were generated by cloning the promoter of PtrCHS1, PtrDRF2 or PtrANS1 into pGreenII0800-LUC [47]. The resultant pGreenII62-SK-PdMYB118, pGreenII62-SKPdTT8 or pGreenII62-SK-PtrJAZ1 was used as effector construct as described above. To detect the inhibition of PtrJAZ1 to the transcription activity of the PdMYB118/ PdTT8 complex, effectors PdMYB118, PdTT8, PdMYB118 + PdTT8 or PdMYB118 + PdTT8 + PtrJAZ1 were repetitively co-expressed with each reporter construct in poplar leaf protoplasts [45]. The LUC/REN ratio was used to represent the relative activity of the transcriptional factors. Three replicates were carried out for each assay, and the variability was indicated with the standard deviation (SD).

\section{Statistical analysis}

All data were obtained from three biological replicates each. For statistical analyses, Student's t-test (two-tailed) or ANOVA (one-way) was used to generate every $P$ value. The variability was indicated with the standard deviation $(\mathrm{SD}) . *{ }^{*} *$ and $* * *$ indicate $p$-values $<0.05,<0.01$ and $<0.001$, respectively.

\section{Supplementary information}

Supplementary information accompanies this paper at https://doi.org/10. 1186/s12870-020-02389-1.

Additional file 1: Figure S1. Wound induced anthocyanin biosynthesis in the leaf of transgenic plants.

Additional file 2: Figure S2. BiFC assays to test the interaction of PtrJAZ1 with PdMYB118.

Additional file 3: Figure S3. Expression of PtrJAZ1 and PdTT8 in the protoplasts isolated from the leaves of transgenic plants overexpressing PdMYB118.

Additional file 4: Figure S4. qRT-PCR analyses of $P d M Y B 118$ and $P d T T 8$ in the wounded leaves of WT and transgenic plants overexpressing PdMYB118.

Additional file 5: Figure S5. A proposed model of wound induced anthocyanin biosynthesis in poplar.

Additional file 6: Figure S6. Western blotting analyses of JAZ1 protein in the wounded leaves.

Additional file 7: Figure S7. Western blotting analyses of JAZ1 protein in the wounded leaves of transgenic poplar plants.

Additional file 8: Table S1. Primer sequences used in this study.

\section{Abbreviations}

TF: Transcription factors; ABG: Anthocyanin biosynthesis gene; MeJA: Methyl jasmonate; JAZ: JASMONATE ZIM-domain; CHS: Chalcone synthase;

$\mathrm{CHI}$ : Chalcone isomerase; F3H: Flavanone 3-hydroxylase; F3'H: Flavanone 3'hydroxylase; F3'5'H: Flavonoid 3'5'-hydroxylase; DFR: Dihydroflavonol 4reductase; ANS: Anthocyanidin synthase; bHLH: Basic helix-loop-helix; WT: Wild type

\section{Acknowledgements}

We thank Prof. Gui-Feng Liu (Northeast Forestry University, China) for kindly providing us the Shanxin yang $(P$. davidiana $\times P$. bolleana) clone.

\section{Authors' contributions \\ $H W, X W, C Y, C W$ and YL performed the experiments and analyzed data. HW and $\mathrm{HZ}$ wrote the manuscript and arranged all data. All authors contributed revision of the manuscript. All authors read and approved the manuscript.}

\section{Funding}

This work was supported by the following grants: the National Key R \& D Program of China (2016YFD0600106, 2019YFD1000500); the National Key Program on Transgenic Research (2018ZX08020002-003-004); the National Natural Science Foundation of China (31700524, 31870576, 31871625); the Modern Agricultural Industry Technology System Innovation Team of Shandong Province of China (SDAIT-02-05); the Agricultural Variety Improvement Project of Shandong Province (2019LZGC009). The funders played no role in the design of the study and collection, analysis, and interpretation of data and in writing the manuscript. 


\section{Availability of data and materials}

All materials and data analyzed in this study are available from the corresponding author

\section{Ethics approval and consent to participate}

This study does not contain any research requiring ethical consent or approval.

\section{Consent for publication}

Not applicable.

\section{Competing interests}

The authors declare that they have no competing interests.

\section{Author details}

${ }^{1}$ National Key Laboratory of Plant Molecular Genetics, Shanghai Institute of Plant Physiology and Ecology, Chinese Academy of Sciences, 300 Fenglin Road, Shanghai 200032, China. ${ }^{2}$ Forestry and Pomology Research Institute, Shanghai Academy of Agriculture Sciences, 1000 Jinqi Road, Shanghai, China. ${ }^{3}$ College of Agriculture, Ludong University, 186 Hongqizhong Road, Yantai 264025, China. ${ }^{4}$ The Key Laboratory of Molecular Module-Based Breeding of High Yield and abiotic Resistant Plants in the Universities of Shandong, Ludong University, 186 Hongqizhong Road, Yantai 264025, China.

Received: 6 February 2020 Accepted: 6 April 2020

Published online: 20 April 2020

\section{References}

1. Winkel-Shirley B. Flavonoid biosynthesis. A colorful model for genetics, biochemistry, cell biology, and biotechnology. Plant Physiol. 2001;126:485-93.

2. Christie PJ, Alfenito MR, Walbot V. Impact of low-temperature stress on general phenylpropanoid and anthocyanin pathways: enhancement of transcript abundance and anthocyanin pigmentation in maize seedlings. Planta. 1994;194:541-9.

3. Sarma AD, Sharma R. Anthocyanin-dna copigmentation complex: mutual protection against oxidative damage. Phytochemistry. 1999;52:1313-8.

4. Steyn W, Wand S, Holcroft D, Jacobs G. Anthocyanins in vegetative tissues: a proposed unified function in photoprotection. New Physiol. 2002;155:349-61.

5. Lorenc-Kukuła K, Jafra S, Oszmiański J, Szopa J. Ectopic expression of anthocyanin 5-o-glucosyltransferase in potato tuber causes increased resistance to bacteria. J Agr Food Chem. 2005;53:272-81.

6. Nakabayashi R, Yonekura-Sakakibara K, Urano K, Suzuki M, Yamada Y Nishizawa T, Matsuda F, Kojima M, Sakakibara H, Shinozaki K, Michael AJ, Tohge T, Yamazaki M, Saito K. Enhancement of oxidative and drought tolerance in Arabidopsis by overaccumulation of antioxidant flavonoids. Plant J. 2014:77:367-79.

7. Tsai CJ, Harding SA, Tschaplinski TJ, Lindroth RL, Yuan YN. Genome-wide analysis of the structural genes regulating defense phenylpropanoid metabolism in Populus. New Physiol. 2006;172:47-62.

8. Mellway RD, Tran LT, Prouse MB, Campbell MM, Constabel CP. The wound-, pathogen-, and ultraviolet B-responsive MYB134 gene encodes an R2R3 MYB transcription factor that regulates proanthocyanidin synthesis in poplar. Plant Physiol. 2009;150:924-41.

9. James AM, Ma D, Mellway R, Gesell A, Yoshida K, Walker V, Tran L, Stewart D, Reichelt M, Suvanto J, Salminen JP, Gershenzon J, Séguin A, Constabel CP. Poplar MYB115 and MYB134 transcription actors regulate proanthocyanidin synthesis and structure. Plant Physiol. 2017;174:154-71.

10. Wang L, Ran L, Hou Y, Tian Q, Li C, Liu R, Fan D, Luo K. The transcription factor MYB115 contributes to the regulation of proanthocyanidin biosynthesis and enhances fungal resistance in poplar. New Phytologist. 2017;215:351-67.

11. Ullah C, Tsai CJ, Unsicker SB, Xue LJ, Reichelt M, Gershenzon J. Hammerbacher A. Salicylic acid activates poplar defense against the biotrophic rust fungus Melampsora larici-populina via increased biosynthesis of catechin and proanthocyanidins. New Physiol 2019a;221:960-975.

12. Ullah C, Unsicker SB, Reichelt M, Gershenzon J. Hammerbacher A. Accumulation of catechin and proanthocyanidins in black poplar stems after infection by Plectosphaerella populi: hormonal regulation, biosynthesis and antifungal activity. Front Plant Sci 2019b;10:1-10.
13. Espley RV, Hellens RP, Putterill J, Stevenson DE, Kutty-Amma S, Allan AC. Red colouration in apple fruit is due to the activity of the MYB transcription factor, MdMYB10. Plant J. 2007;49:414-27.

14. Hancock KR, Collette V, Fraser K, Greig M, Xue H, Richardson K, Jones C, Rasmussen S. Expression of the R2R3-MYB transcription factor TaMYB14 from Trifolium arvense activates proanthocyanidin biosynthesis in the legumes Trifolium repens and Medicago sativa. Plant Physiol. 2012;159:1204-20.

15. Hichri I, Barrieu F, Bogs J, Kappel C, Delrot S, Lauvergeat V. Recent advances in the transcriptional regulation of the flavonoid biosynthetic pathway. J Exp Bot. 2011;62:2465-83.

16. Petroni K, Tonelli C. Recent advances on the regulation of anthocyanin synthesis in reproductive organs. Plant Sci. 2001;181:219-29.

17. Xu W, Dubos C, Lepiniec L. Transcriptional control of flavonoid biosynthesis by MYB-bHLH-WDR complexes. Trends in Plant Sci. 2015;20:176-85.

18. Grotewold E, Sainz MB, Tagliani L, Hernandez JM, Bowen B, Chandler VL. Identification of the residues in the Myb domain of maize $C 1$ that specify the interaction with the bHLH cofactor R. P Natl Acad Sci USA. 2000;97: 13579-84.

19. Elomaa $\mathrm{P}$, Uimari $\mathrm{A}$, Mehto $\mathrm{M}$, Albert VA, Laitinen RA, Teeri TH. Activation of anthocyanin biosynthesis in Gerbera hybrida (Asteraceae) suggests conserved protein-protein and protein-promoter interactions between the anciently diverged monocots and eudicots. Plant Physiol. 2003;133:1831-42

20. Sweeney MT, Thomson MJ, Pfeil BE, McCouch S. Caught red-handed: RC encodes a basic helix-loop-helix protein conditioning red pericarp in rice. Plant Cell. 2006;18:283-94.

21. Nakatsuka T, Haruta KS, Pitaksutheepong C, Abe Y, Kakizaki Y, Yamamoto K, Shimada N, Yamamura S, Nishihara M. Identification and characterization of R2R3-MYB and bHLH transcription factors regulating anthocyanin biosynthesis in gentian flowers. Plant Cell Physiol. 2008:49:1818-29.

22. Matus JT, Poupin MJ, Canon P, Bordeu E, Alcalde JA, Arce-Johnson P. Isolation of WDR and bHLH genes related to flavonoid synthesis in grapevine (Vitis vinifera L.). Plant Mol Biol. 2010;72:607-20

23. Chung HS, Koo AJK, Gao X, Jayanty S, Thines B, Jones AD, Howe GA. Regulation and function of Arabidopsis JASMONATE ZIM-domain genes in response to wounding and herbivory. Plant Physiol. 2008;146:952-64.

24. Koo AJ, Gao X, Jones AD, Howe GA. A rapid wound signal activates the systemic synthesis of bioactive jasmonates in Arabidopsis. Plant J. 2009;59: 974-86.

25. Staswick PE. JAZing up jasmonate signaling. Trends in Plant Sci. 2008;13:66-71.

26. Gfeller A, Dubugnon L, Liechti R, Farmer EE. Jasmonate biochemical pathway. Sci Signal. 2010;3:332

27. Thines B, Katsir L, Melotto M, Niu Y, Mandaokar A, Liu G, Nomura K, He SY, Howe GA, Browse J. JAZ repressor proteins are targets of the SCF (COI1) complex during jasmonate signalling. Nature. 2007;448:661-5.

28. Gan Y, Li H, Xie Y, Wu W, Li M, Wang X, Huang J. THF1 mutations lead to increased basal and wound-induced levels of oxylipins that stimulate anthocyanin biosynthesis via COI1 signaling in Arabidopsis. J Integr Plant Biol. 2014:56:916-27.

29. Qi T, Song S, Ren Q, Wu D, Huang H, Chen Y, Fan M, Peng W, Ren C, Xie D. The Jasmonate-ZIM-domain proteins interact with the WD-repeat/bHLH/ MYB complexes to regulate jasmonate-mediated anthocyanin accumulation and trichome initiation in Arabidopsis thaliana. Plant Cell. 2011:23:1795-814.

30. Xie C, Wang D, Kou F, Kang D, Yang X. The pathogen and wound induces expression of genes related to proanthocyanidins (PAs) synthesis in cotton leaves. Am J Plant Sci. 2012;3:228-34.

31. An J, Zhang $X$, You C, Bi S, Wang $X$, Hao Y. MdWRKY40 promotes wounding-induced anthocyanin biosynthesis in association with MdMYB1 and undergoes MdBT2-mediated degradation. New Phytol. 2019;224:380-95.

32. Wilkins O, Nahal H, Foong J, Provart NJ, Campbell MM. Expansion and diversification of the Populus R2R3-MYB family of transcription factors. Plant Physiol. 2009;149:981-93.

33. Cho JS, Nguyen VP, Jeon HW, Kim MH, Eom SH, Lim YJ, Kim WC, Park EJ, Choi YI, Ko JH. Overexpression of PtrMYB119, a R2R3-MYB transcription factor from Populus trichocarpa, promotes anthocyanin production in hybrid poplar. Tree Physiol. 2016;36:1162-76.

34. Wang $H H$, Wang $X Q$, Song $W M$, Bao $Y$, Jin $Y L$, Jiang $C M$, Wang $C T$, Li $B$, Zhang HX. PdMYB118, isolated from a red leaf mutant of Populus deltoids, is a new transcription factor regulating anthocyanin biosynthesis in poplar. Plant Cell Rep. 2019;38:927-36. 
35. Sheard LB, Tan X, Mao H, Withers J, Ben-Nissan G, TR RH, Kobayashi Y, Hsu FF, Sharon M, Browse J, He SY, Rizo J, Howe GA, Zheng N. Jasmonate perception by inositol-phosphate-potentiated COI1-JAZ co-receptor. Nature. 2010:468:400-5.

36. An XH, Tian Y, Chen KQ, Liu XJ, Liu DD, Xie XB, Cheng CG, Cong PH, Hao YJ. MdMYB9 and MdMYB11 are involved in the regulation of the JA-induced biosynthesis of anthocyanin and proanthocyanidin in apples. Plant Cell Physiol. 2015;56:650-62.

37. Tamari G, Borochov A, Atzorn R, Weiss D. Methyl jasmonate induces pigmentation and flavonoid gene expression in petunia corollas: A possible role in wound response. Physiol Plantarum. 1995;94:45-50.

38. Nesi N, Jond C, Debeaujon I, Caboche M, Lepiniec L. The Arabidopsis TT2 gene encodes an R2R3 MYB domain protein that acts as a key determinant for proanthocyanidin accumulation in developing seed. Plant Cell. 2001;13: 2099-114.

39. Quattrocchio F, Verweij W, Kroon A, Spelt C, Mol J, Koes R. PH4 of petunia is an R2R3 MYB protein that activates vacuolar acidification through interactions with basic-helix-loop-helix transcription factors of the anthocyanin pathway. Plant Cell. 2006;18:1274-91.

40. Hichri I, Heppel SC, Pillet J, Léon C, Czemmel S, Delrot S, Lauvergeat V, Bogs J. The basic helix-loop-helix transcription factor MYC1 is involved in the regulation of the flavonoid biosynthesis pathway in grapevine. Mol Plant. 2010;3:509-23.

41. Wasternack C, Song S. Jasmonates: biosynthesis, metabolism, and signaling by proteins activating and repressing transcription. J Exp Bot. 2017;68:1303-21.

42. Loreti E, Povero G, Novi G, Solfanelli C, Alpi A, Perata P. Gibberellins, jasmonate and abscisic acid modulate the sucrose-induced expression of anthocyanin biosynthetic genes in Arabidopsis. New Phytol. 2008;179:1004-16.

43. Shan X, Zhang Y, Peng W, Wang Z, Xie D. Molecular mechanism for jasmonate-induction of anthocyanin accumulation in Arabidopsis. J Exp Bot. 2009;60:3849-60.

44. Wang HH, Wang $C T$, Liu H, Tang RJ, Zhang HX. An efficient agrobacteriummediated transformation and regeneration system for leaf explants of two elite aspen hybrid clones Populus alba $\times$ P. berolinensis and Populus davidiana X P. bolleana. Plant Cell Rep. 2011;30:2037-44.

45. Wang HH, Tang RJ, Liu H, Chen HY, Liu JY, Jiang XN, Zhang HX. Chimeric repressor of PtSND2 severely affects wood formation in transgenic Populus. Tree Physiol. 2013;33:878-86.

46. Yoshida K, Ma D, Constabel CP. The MYB182 protein down-regulates proanthocyanidin and anthocyanin biosynthesis in poplar by repressing both structural and regulatory flavonoid genes. Plant Physiol. 2015;167:693-710.

47. Wang HH, Jiang $C M$, Wang $C T$, Yang $Y$, Gao XY, Zhang HX. Antisense expression of the fasciclin-like arabinogalactan protein PtFLA6 gene in Populus inhibits expression of its homologous genes and alters stem biomechanics and cell wall composition in transgenic trees. J Exp Bot. 2015; 66:1291-302.

48. Chen ML, Fu XM, Liu JQ, Ye TT, Hou SY, Huang YQ, Yuan BF, Wu Y, Feng YQ. Highly sensitive and quantitative profiling of acidic phytohormones using derivatization approach coupled with nano-LC-ESI-Q-TOF-MS analysis. J Chromatogr B. 2012:905:67-74.

49. Hellens RP, Allan AC, Friel EN, Bolitho K, Grafton K, Templeton MD, Karunairetnam S, Gleave AP, Laing WA. Transient expression vectors for functional genomics, quantification of promoter activity and RNA silencing in plants. Plant Methods. 2005;1:13.

\section{Publisher's Note}

Springer Nature remains neutral with regard to jurisdictional claims in published maps and institutional affiliations.

Ready to submit your research? Choose BMC and benefit from:

- fast, convenient online submission

- thorough peer review by experienced researchers in your field

- rapid publication on acceptance

- support for research data, including large and complex data types

- gold Open Access which fosters wider collaboration and increased citations

- maximum visibility for your research: over $100 \mathrm{M}$ website views per year

At BMC, research is always in progress.

Learn more biomedcentral.com/submissions 